\title{
Détermination de la flore bactérienne caséolytique psychrotrophe des laits crus réfrigérés
}

\author{
par \\ MILLIERE (J.B.)* et VEILLET-PONCET (L.)**
}

Après avoir dénombré, dans un précédent article [78], les flores aérobies psychrotrophe et mésophile, possédant ou non une activité protéolytique, isolées de 50 échantillons de lait cru réfrigéré en tanks à la ferme, nous nous proposons d'identifier la flore caséolytique psychrotrophe.

Les recherches relatives à la fréquence et à la caractérisation des bactéries psychrotrophes qui élaborent les protéases étant relativement rares, nous comparerons nos résultats avec les divers travaux consacrés à l'étude de la flore aérobie psychrotrophe.

\section{MATERIEL ET METHODES}

Pendant la période du 16 mai au 6 juin 1975, 50 échantillons de lait cru réfrigéré sont collectés dans 50 fermes situées au sud-ouest de Nancy. Chaque échantillon de $150 \mathrm{ml}$, prélevé dans un flacon en verre de $250 \mathrm{ml}$, stérile, et entreposé pendant le transport dans une glacière à $4^{\circ} \mathrm{C}$, est étudié dès son arrivée au laboratoire de bactériologie.

Les dilutions décimales sont réalisées à l'aide de Tryptone-sel (T.S.) et les numérations de la flore caséolytique psychrotrophe sont effectuées sur milieu gélosé au lait, milieu Tryptone-Agar $\left(\mathrm{A}_{1}\right)$ préconisé par la F.I.L. (composition en g/1 : tryptone, 5 ; extrait de levures, 2,5 ; glucose, 1 ; agar, 15 ; lait écrémé, $10 \mathrm{ml}$ ), incubé à $7 \pm 0,5^{\circ} \mathrm{C}$ pendant 10 jours (A.P.H.A. [2]).

\footnotetext{
* I.U.T. de Biologie Appliquée - 54600 Villers-les-Nancy.

** Laboratoire de Microbiologie Alimentaire, E.N.S.A.I.A.-I.N.P.L., 32, rue SainteCatherine - 54000 Nancy.
} 


\section{Isolement des clones}

Pour l'échantillon correspondant à chaque producteur, la culture en boîte de Pétri, retenue pour le repiquage des souches caséolytiques, comporte de 30 à 300 colonies : 20 colonies isolées sont prélevées dans un secteur et repiquées dans $10 \mathrm{ml}$ de bouillon nutritif (B.N.), puis sont incubées pendant 24 à $48 \mathrm{~h}$, à $25^{\circ} \mathrm{C}$.

L'ensemencement en stries d'un milieu gélosé au lait $\left(\mathrm{A}_{2}\right)$ (composition en g/1 : lait écrémé Spray, 50 ; Agar, 15), coulé en boîtes de Pétri, permet, après incubation à $25^{\circ} \mathrm{C}$ pendant $72 \mathrm{~h}$, le contrôle de la pureté de la souche, la vérification de l'activité protéolytique et l'estimation de cette activité par la mesure du diamètre de la zone d'éclaircissement entourant la colonie bactérienne.

L'acidification du lait pouvant produire de fausses réactions, il s'avère indispensable, après repiquage de la colonie sur gélose nutritive inclinée (G.N.I.), d'inonder la surface de la gélose au lait avec $\mathrm{HCl}$ dilué ou T.C.A dilué, afin de vérifier la persistance de la protéolyse [49].

Après incubation à $25^{\circ} \mathrm{C}$ pendant $24 \mathrm{~h}$, les cultures sont stockées à $+5^{\circ} \mathrm{C}$ jusqu'à leur identification.

Certaines souches, présentant sur G.N.I. un développement faible ou une absence de croissance, sont cultivées sur un milieu gélosé $\left(A_{3}\right)$ riche en facteurs de croissance (composition en $\mathrm{g} / \mathrm{l}$ : bactocasitone (Difco), 5 ; extrait de levures, 2,5 ; glucose, 1 ; lait écrémé, $5 \mathrm{ml}$; agar, $15 ; \mathrm{pH}$ 6,6-7,0). Ce milieu, qui donne entière satisfaction, est utilisé lors des repiquages bimestriels de toutes les souches.

\section{Identification des bactéries caséolytiques}

Avant de procéder à toute identification, les souches sont repiquées sur milieu gélosé $\left(A_{3}\right)$ et incubées à $25^{\circ} \mathrm{C}$ pendant $24 \mathrm{~h}$.

L'identification des clones est conduite d'après les caractères morphologiques, physiologiques et biochimiques proposés d'une part

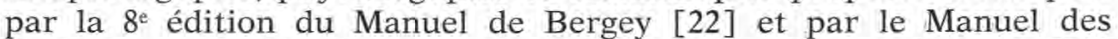
Techniques Bactériologiques de Buttiaux, Beerens et Taquet [18] et, d'autre part, pour certains genres et espèces, à partir des travaux originaux de divers chercheurs et de nos propres investigations.

\subsection{Tests COMmuns a tOUTES LES SOUCHES}

Lors de la purification des souches, l'observation du développement bactérien en B.N., en tube $18 \times 180$, nous renseigne sur le type respiratoire (aérobie strict ou aéro-anaérobie facultatif), sur la nature du trouble (homogène ou granuleux) et sur la pigmentation d'un éventuel sédiment. La morphologie des colonies, leur aspect $\mathrm{S}$ ou $\mathrm{R}$ sur gélose au lait, la présence ou l'absence d'une pigmentation diffu- 
sible ou non et le diamètre de la zone de protéolyse sont également notés.

Certains tests fondamentaux permettent un classement de nos souches en grands groupes, en vue de faciliter leur identification ultérieure :

- la coloration de Gram, modifiée par Burke-Kopelov, permet de séparer les bactéries Gram positives des bactéries Gram négatives ;

- l'observation microscopique du corps bactérien nous renseigne sur la morphologie ;

- la recherche de la catalase ;

- la mise en évidence d'une oxydase (méthode de Kovacs, 1956) ;

- la présence d'une cytochrome-oxydase (réaction de Gaby et Hadley, modifiée par Ewing et Johnson);

- l'étude de la mobilité (sur gélose molle à 5 p. 1000 d'Agar).

\subsection{TESTS PHysiologiQUES ET BIOCHIMIQUES}

\subsubsection{Identification des bactéries Gram positives}

Toutes les souches Gram positives isolées qui, après incubation à $7^{\circ} \mathrm{C}$ pendant $10 \mathrm{j}$, se présentent sous forme de cocci et possèdent une catalase très active, appartiennent à la famille des Micrococcaceae.

La distinction entre les genres Micrococcus et Staphylococcus est fondée sur le type respiratoire et sur le mode de dégradation du glucose, par voie oxydative ou par voie fermentative.

L'identification des sous-groupes ou des espèces du genre Micrococcus est conduite d'après les travaux de Baird-Parker [5], l'étude de Forbes [30] et le Manuel de Bergey [22] ; elle repose sur la production d'acide à partir d'hydrates de carbone (arabinose, lactose, maltose, mannitol, xylose), l'hydrolyse de l'amidon, la production d'acétoïne (technique d'O'Meara), la mise en évidence d'une nitrate-réductase, d'une phosphatase et d'une estérase (Tween 80).

\subsubsection{Identification des bactéries Gram négatives}

Compte tenu des connaissances acquises concernant les bactéries Gram négatives, en forme de bâtonnets ou de coccobacilles [98, 100 , 107], nous établissons un classement de nos souches, fondé sur le mode de dégradation du glucose (milieu de Hugh et Leifson [47], sur la pigmentation observée par culture sur milieu gélosé au lait (pigments diffusibles ou de nature caroténoïde) et sur la réponse au test de l'oxydase :

- Bactéries fermentantes.

- Bactéries oxydantes :

- bactéries fluorescentes ;

- bactéries non fluorescentes, apigmentées ; 
TABLEAU 1

\begin{tabular}{|c|c|c|c|c|c|}
\hline \multirow{2}{*}{ Tests biochimiques } & \multirow{2}{*}{$\begin{array}{c}\text { B. } \\
\text { fermentantes }\end{array}$} & \multicolumn{3}{|c|}{ Bactéries oxydantes } & \multirow{2}{*}{$\begin{array}{c}\text { Bactéries } \\
\text { inertes } \\
\text { ou } \\
\text { alcalinisantes }\end{array}$} \\
\hline & & $\begin{array}{c}\text { B. } \\
\text { fluorescentes }\end{array}$ & $\begin{array}{l}\text { B. non } \\
\text { fluorescentes }\end{array}$ & $\begin{array}{c}\text { B. de couleur } \\
\text { orange ou jaune }\end{array}$ & \\
\hline Fermentations sucrées (Kligler) & + & - & + & + & - \\
\hline$\beta$-galactosidase (Le Minor et Ben Hamida) & + & - & - & + & - \\
\hline A.M.C. - R.M. (milieu de Fouad) & + & - & - & + & + \\
\hline Tryptophane désaminase (réactif Ehrlich-Kovacs) & + & - & + & + & + \\
\hline Amylase, gélatinase ((Frazier) & + & + & + & + & + \\
\hline Nitrate-réductase, uréase (Christensen) & + & + & + & + & + \\
\hline Arginine dihydrolase (A.D.H.) (Thornley) [123] & + & + & + & + & + \\
\hline Estérase, lipase & + & + & + & + & + \\
\hline Lécithinase & - & + & + & + & + \\
\hline Déoxyribonucléase (DNase) & + & - & - & + & + \\
\hline
\end{tabular}


- bactéries élaborant un pigment caroténoïde de couleur orange ou jaune.

- Bactéries inertes et alcalinisantes, apigmentées.

Nous regroupons dans le tableau 1 les principaux tests biochimiques retenus pour l'identification des bactéries Gram négatives.

\subsubsection{Bactéries fermentantes}

Outre les tests précédemment décrits, la dégradation du sorbitol et la recherche d'une O.D.C. (technique de Möller) permettent de différencier, dans la famille des Enterobacteriaceae, les genres de la tribu des Klebsielleae.

Les espèces du genre Aeromonas sont identifiées d'après les caractères biochimiques retenus par Shewan et Véron [22], Kleeberger [59] et Veillet-Poncet [124] ; l'étude des fermentations sucrées (arabinose, galactose, glucose, glycérol, saccharose) est réalisée en milieu liquide contenant une cloche de Durham, avec une concentration finale en hydrate de carbone de 10 p. 100 . La transformation des acides aminés (arginine, lysine, ornithine) est conduite selon la technique de Möller. La production d'A.M.C., à partir de 2-3 butanediol, est mise en évidence par la technique d'O'Meara, en milieu liquide (composition en $\mathrm{g} / 1$ : protéose-peptone, 0,$5 ; \mathrm{K}_{2} \mathrm{HPO}_{4}, 0,5 ; 2-3$ butanediol, 5 ; pH 6, stérilisation par filtration).

\subsubsection{Bactéries oxydantes}

\section{- Bactéries fluorescentes}

Nous regroupons toutes les bactéries qui élaborent, sur milieu gélosé au lait $\left(A_{2}\right)$, un pigment vert diffusible ou pyoverdine, décelable à l'œil nu en lumière naturelle ou dont l'observation est facilitée en lumière de Wood (360 nm).

L'identification est réalisée d'après les travaux de divers chercheurs $[45,65,68]$ et notamment d'après ceux de Stanier et al. [108], Shewan et al. [107]; elle fait appel à quelques tests particuliers : dégradation par voie oxydative, sur milieu de Hugh et Leifson, de l'adonitol, de l'arabinose, de l'inositol et du tréhalose ; production de levane à partir de saccharose (composition en g/1 : saccharose, 40 ; Agar, 12 ; bouillon nutritif $1000 \mathrm{ml}, \mathrm{pH} \mathrm{7,2)} \mathrm{;} \mathrm{recherche} \mathrm{d'autres}$ pigments sur milieu de King, Ward et Raney (1954) ; élaboration d'une lécithinase ; croissance à $4^{\circ} \mathrm{C}$ et $41^{\circ} \mathrm{C}$ en B.N.

\section{- Bactéries non fluorescentes, apigmentées}

Les cultures à $25^{\circ} \mathrm{C}$ sur milieu de King $\mathrm{B}$ et l'observation journalière, pendant 10 jours, du développement bactérien, en lumière de Wood, permettent de vérifier l'absence de production de pyoverdine. Nous utilisons, pour ces souches, les tests précédemment décrits pour l'étude des bactéries fluorescentes. 
- Bactéries non fluorescentes, pigmentées

\section{- A pigment caroténö̈de de couleur orange}

Le milieu de Hugh et Leifson n'est pas approprié à l'étude de ces bactéries. Dégradant le glucose en aérobiose pour 2/3 d'entre elles, nous les classons parmi les bactéries oxydantes.

Leur identification est fondée sur un certain nombre de tests biochimiques particuliers : production de levane à partir de saccharose (selon Graham et Hodgkiss [37]) ; développement sur milieu de Mac Conkey (glucose-Agar) ; croissance sur milieu minimum (composition en g/l : $\mathrm{Mg} \mathrm{SO}_{4}, 7 \mathrm{H}_{2} \mathrm{O}, 0,2 ; \mathrm{NaCl}, 5 ; \mathrm{NH}_{4} \mathrm{Cl}, 1$; glucose, 5 ; solution de bleu de bromothymol à 1,6 p. $100,5 \mathrm{ml} ; \mathrm{pH} \mathrm{7,0)}$; croissance à $41^{\circ} \mathrm{C}$ en B.N., ainsi qu'à des concentrations variant entre 1 et 3 p. 100 en $\mathrm{NaCl}$; variation de la pigmentation de la culture en fonction des barèmes d'incubation $\left(7^{\circ} \mathrm{C} / 10 \mathrm{j} ; 25^{\circ} \mathrm{C} / 4 \mathrm{j} ; 37^{\circ} \mathrm{C} / 2 \mathrm{j}\right.$ ) ; recherches d'une phénylalanine désaminase, d'une phosphatase, de lipases (Tween 80 et Tween 85), d'hémolysine (sang de mouton); hydrolyse de l'esculine, sensibilité à la polymyxine $\mathrm{B}$, en disques à $50 \mathrm{U}$ et à $300 \mathrm{U}$; mise en évidence d'une mobilité par glissement due à des mouvements de reptation ou d'ondulation sur milieu gélosé de Hendrie [18] (composition en g/l : extrait de levures, 1 ; Ionagar $\mathrm{n}^{\circ} 2$ (Oxoïd), 8 ; pH 7-7,4) et de Hayes [40] (composition en g/1 : LabLemco, 1 ; $\mathrm{NaCl}, 2,5$; Agar, 15 ; additions de concentrations variables en peptone, de 1 à $10 \mathrm{~g}$ ); recherche d'une activité cellulolytique [32, 126], soit par culture sur disque de papier filtre Durieux, de $\varnothing 9 \mathrm{~cm}$, déposé sur milieu minéral gélosé (composition en $\mathrm{g} / 1: \mathrm{K}_{2} \mathrm{HPO}_{4}, 1$; $\mathrm{KNO}_{3}, 1 ; \mathrm{MgSO}_{4}, 0,5 ; \mathrm{CaCl}_{2}, 0,2 ; \mathrm{FeCl}_{3}$, deux gouttes ; gélose, 20 ; $\mathrm{pH} 7-7,5)$ soit par immersion partielle de bandelettes de papier filtre, en position verticale, dans un milieu semi-liquide de composition identique, mais contenant en plus 0,05 p. 100 d'extrait de levures et gélosé à 1,2 p. 1000 ; dans ce cas, la présence d'enzymes cellulolytiques se traduit soit par la présence de trous, soit par l'accumulation d'un sédiment au fond du tube.

\section{- A pigment caroténoïde jaune}

Nous retenons pour ce groupe de bactéries la clef d'identification proposée par Gilardi en 1975 [35].

\section{- Bactéries inertes et alcalinisantes, apigmentées}

Le mode de dégradation du glucose sur milieu de Hugh et Leifson et la recherche d'une oxydase permettent de différencier les Pseudomonas du groupe III de Shewan et al. [107], de ceux du groupe IV, et des Acinetobacter. L'identification de l'espèce A. calcaoceticus est fondée sur quelques tests complémentaires : développement à $42^{\circ} \mathrm{C}$; croissance en présence de 2,5 p. 100 de $\mathrm{NaCl}$; production $\mathrm{d}^{\prime} \mathrm{H}_{2} \mathrm{~S}$; recherche de la sensibilité à la polymyxine $\mathrm{B}$ en disques à $50 \mathrm{U}$ et à la pénicilline ; mise en évidence d'une hémolysine (sang de mouton). 


\section{RESULTATS}

Les taux de contamination des laits crus en bactéries psychrotrophes caséolytiques traduisent une grande hétérogénéité dans les soins apportés lors de la traite et du stockage du lait, car ils varient entre $3.10^{2}$ et $6,4.10^{5}$ bactéries par $\mathrm{ml}: 27$ et 9 prélèvements contiennent respectivement plus de $1.10^{4}$ et plus de $1.10^{5}$ bactéries par $\mathrm{ml}$ $[77,78]$.

\section{Répartition des bactéries selon les principaux groupes rencontrés}

Le tableau 2 rend compte des groupes de bactéries isolées.

\section{Identification des bactéries Gram positives}

Dans le tableau 3 sont consignées les espèces de Micrococcaceae mises en évidence.

\section{Identification des bactéries Gram négatives}

\subsection{BACTÉRIES FERMENTANTES}

Le tableau 4 permet de différencier les bactéries fermentantes selon leur réponse au test de l'oxydase.

\subsection{BACTÉRIES OXYDANTES ET ALCALINISANTES}

Le tableau 5 classe les bactéries oxydantes et alcalinisantes d'après leur pigmentation.

\section{Tableaux synoptiques}

4.1. COMPOSITION DE LA FLORE CASÉOLYTIQUE PSYCHROTROPHE ISOLÉE DE 50 ÉCHANTILLONS DE LAIT CRU

Dans le tableau 6 sont regroupées les espèces caséolytiques psychrotrophes isolées.

4.2. PROPORTION DES DIFFÉRENTES ESPĖCES DANS LA FLORE CASÉOLYTIQUE

Le tableau 7 traduit la contamination des laits crus et indique la fréquence des différentes espèces dans les échantillons.

Nous remarquons que 10 échantillons possèdent une flore caséolytique psychrotrophe composée uniquement de Cytophaga, alors que dans 7 laits, ce genre est absent.

\subsection{Espèces caséolytiques pigmentées}

Le tableau 8 rend compte du classement des espèces selon leur pigmentation sur milieu gélosé au lait $\left(A_{2}\right)$. 
TABLEAU 2

\begin{tabular}{|c|c|c|c|c|c|c|c|}
\hline \multirow{3}{*}{$\begin{array}{c}\text { Bactéries } \\
\text { Gram } \\
\text { positives }\end{array}$} & \multicolumn{6}{|c|}{ Bactéries Gram négatives (963 souches) } & \multirow{3}{*}{$\begin{array}{c}\text { Total } \\
\text { des } \\
\text { bactéries }\end{array}$} \\
\hline & \multicolumn{2}{|c|}{ B. fermentantes } & \multicolumn{2}{|c|}{ B. oxydantes } & \multicolumn{2}{|c|}{ B. alcalinisantes } & \\
\hline & Oxydase + & Oxydase - - & Oxydase + & Oxydase - & Oxydase + & Oxydase - & \\
\hline 33 & 35 & 62 & 855 & 0 & 0 & 11 & 996 \\
\hline
\end{tabular}

TABLEAU 3

\begin{tabular}{|c|c|c|c|}
\hline & M. luteus & M. varians & Nombre total \\
\hline Nombre de souches & 26 & 7 & 33 \\
\hline Nombre d'échantillons contaminés & 4 & 4 & $7^{*}$ \\
\hline
\end{tabular}

* Un échantillon est contaminé par les deux espèces. 


\section{TABLEAU 4}

\begin{tabular}{|c|c|c|c|c|c|c|c|}
\hline & \multicolumn{3}{|c|}{ Oxydase positive } & \multirow{2}{*}{ Total } & \multicolumn{2}{|c|}{ Oxydase négative } & \multirow{2}{*}{ Total } \\
\hline & A. caviae & A. hydrophila & A. punctata & & S. marcescens & E. cloacae & \\
\hline Nombre de souches & 23 & 7 & 5 & 35 & 61 & 1 & 62 \\
\hline Nombre d'échantillons contaminés & 4 & 3 & 2 & $4^{*}$ & 8 & 1 & $8^{*}$ \\
\hline
\end{tabular}

* Certains échantillons sont contaminés par une ou plusieurs espèces.

TABLEAU 5

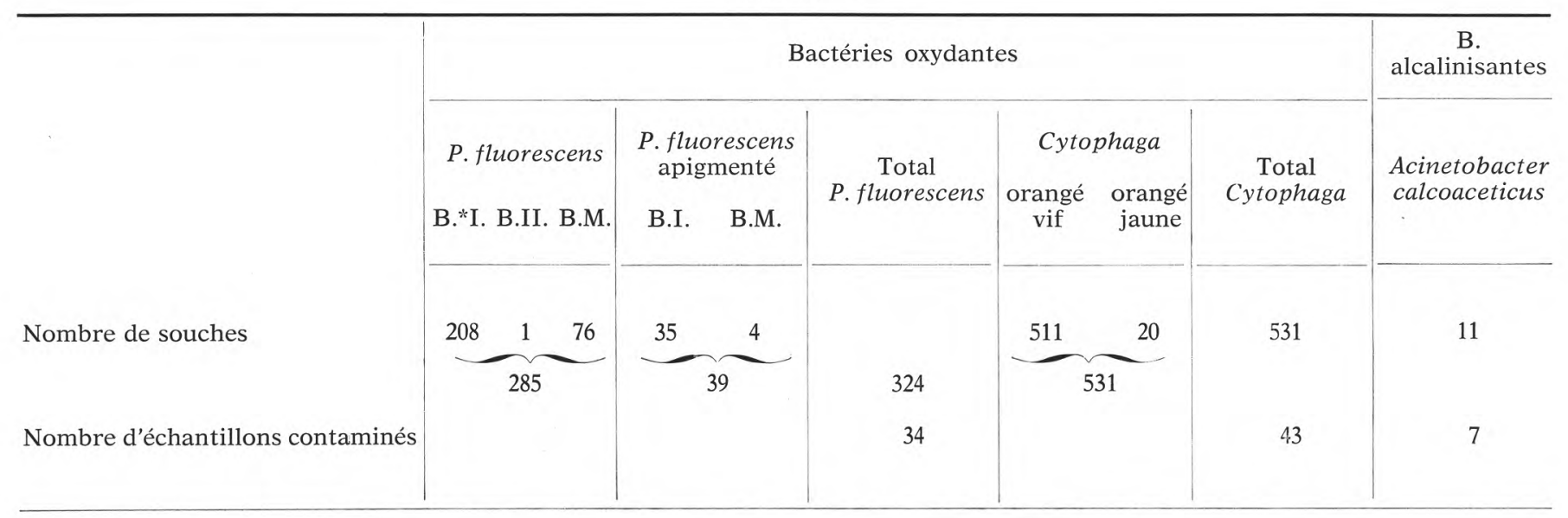

B.* = biotype. 
TABLEAU 6

\begin{tabular}{|c|c|c|c|c|c|c|c|}
\hline & \multirow{2}{*}{$\begin{array}{c}\begin{array}{c}\text { Bactéries } \\
\text { Gram } \\
\text { positives }\end{array} \\
\text { Micrococcus }\end{array}$} & \multicolumn{5}{|c|}{ Bactéries Gram négatives } & \multirow{2}{*}{$\begin{array}{c}\text { Total } \\
\text { des } \\
\text { souches }\end{array}$} \\
\hline & & Cytophaga & P. fluorescens & $\begin{array}{l}\text { S. marcescens } \\
\text { E. cloacae }\end{array}$ & Aeromonas & $\begin{array}{l}\text { Acinetobacter } \\
\text { calcoaceticus }\end{array}$ & \\
\hline $\begin{array}{l}\text { Nombre } \\
\text { de souches }\end{array}$ & 33 & 531 & 324 & 62 & 35 & 11 & 996 \\
\hline p. 100 & 3,31 & 53,31 & 32,53 & 6,22 & 3,51 & 1,10 & \\
\hline
\end{tabular}


TABLEAU 7

\begin{tabular}{|c|c|c|c|c|c|c|c|c|c|c|c|c|c|}
\hline \multirow{2}{*}{ Espèces } & \multirow{2}{*}{$\begin{array}{c}\text { Nombre } \\
\text { d'échantil- } \\
\text { lons non } \\
\text { contaminés }\end{array}$} & \multicolumn{11}{|c|}{ Nombre d'échantillons contaminés dans chaque classe } & \multirow{2}{*}{$\begin{array}{c}\text { Total } \\
\text { d'échantil- } \\
\text { lons } \\
\text { contaminés }\end{array}$} \\
\hline & & $\begin{array}{c}1-10 \\
\text { p. } 100\end{array}$ & $\begin{array}{l}10-20 \\
\text { p. } 100\end{array}$ & $\begin{array}{l}20-30 \\
\text { p. } 100\end{array}$ & $\begin{array}{l}30-40 \\
\text { p. } 100\end{array}$ & $\begin{array}{l}40-50 \\
\text { p. } 100\end{array}$ & $\begin{array}{c}50-60 \\
\text { p. } 100\end{array}$ & $\begin{array}{l}60-70 \\
\text { p. } 100\end{array}$ & $\begin{array}{l}70-80 \\
\text { p. } 100\end{array}$ & $\begin{array}{l}80-90 \\
\text { p. } 100\end{array}$ & $\begin{array}{l}90-99 \\
\text { p. } 100\end{array}$ & $\begin{array}{l}99-100 \\
\text { p. } 100\end{array}$ & \\
\hline Cytophaga & 7 & 4 & 2 & 3 & 5 & 2 & 5 & 4 & 3 & 4 & 1 & 10 & 43 \\
\hline P. fluorescens & 16 & 4 & 7 & 2 & 3 & 3 & 3 & 3 & 3 & 1 & 1 & 4 & 34 \\
\hline Acinetobacter & 43 & 7 & & & & & & & & & & & 7 \\
\hline $\left.\begin{array}{ll}\text { S. } \text { marcescens } \\
\text { E. } \text { cloacae }\end{array}\right\}$ & 42 & 3 & 1 & 1 & & & 1 & & & & 2 & & 8 \\
\hline Aeromonas & 46 & & 2 & & & & 1 & & & 1 & & & 4 \\
\hline Micrococcus & 43 & 2 & 2 & 1 & 1 & 1 & & & & & & & 7 \\
\hline
\end{tabular}


TABLEAU 8

\begin{tabular}{|c|c|c|c|c|c|c|}
\hline \multirow[b]{2}{*}{ Coloration } & \multirow{2}{*}{$\begin{array}{l}\text { Pigment diffusible } \\
\text { vert (pyoverdine) }\end{array}$} & \multicolumn{3}{|c|}{ Pigment non diffusible } & \multirow{2}{*}{$\begin{array}{c}\text { Total } \\
\text { souches } \\
\text { pigmentées }\end{array}$} & \multirow{2}{*}{$\begin{array}{c}\text { p. } 100 \\
\text { souches } \\
\text { pigmentées }\end{array}$} \\
\hline & & jaune vif & $\begin{array}{c}\text { coloration } \\
\text { orange }\end{array}$ & rouge & & \\
\hline Espèces & P. fluorescens & $\begin{array}{l}\text { M. luteus } \\
\text { M. varians }\end{array}$ & Cytophaga & S. marcescens & & \\
\hline Nombre de souches & 285 & 16 & 531 & 3 & 835 & 83,8 \\
\hline
\end{tabular}




\section{DISCUSSION}

Les travaux sur l'identification de la flore caséolytique psychrotrophe des laits crus étant relativement rares $[10,58]$, nous nous référons aux nombreux résultats concernant la flore aérobie psychrotrophe, car elle est constituée, dans la plupart des études, en majorité de souches caséolytiques $[10,51,58,74,75,87,105]$. Thomas et Thomas, en 1973 [122], indiquent que 51 à 97 p. 100 des souches psychrotrophes isolées de laits crus sont fortement protéolytiques et Schultze et Olson [105] rapportent que 90 p. 100 des 586 cultures de bactéries psychrotrophes isolées de laits ou de produits laitiers sont soit protéolytiques, soit lipolytiques et que 66 p. 100 possèdent ces deux activités.

\section{Bactéries Gram positives}

Les 33 microcoques identifiés, $M$. varians et $M$. luteus, peuvent être classés respectivement dans les sous-groupes 5 et 7 et BairdParker [5]. Dans une étude portant sur 39 échantillons de laits crus, Willsens [129] identifie essentiellement le sous-groupe 5 (75 p. 100 des analyses), puis les sous-groupes 6 et 7, dans 20 p. 100 des cas.

Le genre Micrococcus ne constitue, quant à nous, que 3,3 p. 100 de la flore caséolytique psychrotrophe et est absent dans 43 prélèvements.

Ce genre renferme des espèces généralement protéolytiques [24] et, d'après les résultats de certains travaux, il peut représenter en moyenne jusqu'à 22,8 p. 100 [89] ou 27 p. 100 [91] de la flore aérobie psychrotrophe. Cependant, cette proportion, souvent comprise entre 4 et 10 p. $100[3,11,48,90]$, peut être inférieure à 3 p. $100[23,76,84]$. Cette faible teneur en bactéries Gram positives semble être en relation avec une contamination importante de la plupart des échantillons étudiés, car généralement dans les laits paucimicrobiens, ces bactéries, et plus particulièrement le genre Micrococcus, dominent [9, $13,14]$; cependant, nous n'avons pu confirmer cette observation.

\section{Bactéries Gram négatives}

Dans notre étude, 96,7 p. 100 de la flore caséolytique psychrotrophe sont constitués par des bactéries Gram négatives. Dans la flore aérobie psychrotrophe, des proportions encore plus élevées sont rapportées : 98,5 p. 100 par Thomas et Sekhar [120] et 100 p. 100 par Schultze et Olson [104, 105].

92,4 p. 100 des souches Gram négatives possèdent une oxydase très active et 88,8 p. 100 sont capables d'oxyder le glucose. La plupart des bactéries psychrotrophes ne dégradent pas activement les hydrates de carbone et sont considérées comme relativement inertes [80]. 
Seules les bactéries fermentantes (9,7 p. 100), essentiellement les espèces du genre Aeromonas et de la famille des Enterobacteriaceae produisent abondamment de l'acide [118], même à basse température [38] et parfois du gaz à partir de nombreux sucres.

\subsection{BACTÉRIES FERMENTANTES}

Si, dans notre étude, les bactéries coliformes caséolytiques ne représentent en moyenne que 6,2 p. 100 de la flore psychrotrophe, leur proportion peut cependant varier de 0 à 100 p. 100 selon les échantillons.

Quelques publications rapportent le caractère psychrotrophe de certaines bactéries coliformes ; elles se multiplient lentement à des températures comprises entre 1,5 et $5^{\circ} \mathrm{C}[26,31,72,95,117,125]$, et plus activement à $7^{\circ} \mathrm{C}$ [95], mais ne prédominent jamais à des températures de stockage inférieures à $10^{\circ} \mathrm{C}$.

Le genre Klebsiella $[31,73]$, avec l'espèce $K$. aerogenes $[38,94]$, ainsi que l'espèce E. coli (94), sont souvent citées, mais en moyenne, les bactéries coliformes ne représentent que 4 à 20 p. 100 de la flore aérobie psychrotrophe $[3,62,69,90,114]$. Ce groupe de bactéries n'hydrolyse généralement pas la caséine [43], car seulement 7 p. 100 des 1138 bactéries coliformes [118] et $1 / 3$ des $K$. cloacae [111], isolées de laits, possèdent des enzymes protéolytiques. Cependant, quelques espèces protéolysent activement le lait, telles $K$. aerogenes (ou $A$. aerogenes), devenue $K$. pneumoniae $[64,95,106,110,116], E$. cloacae $[64,95,110]$, E. liquefaciens (A. liquefaciens), espèce non pigmentée qui peut être assimilée au genre Serratia $[106,110,116]$ et S. marcescens $[4,64,102,119]$.

Il n'est donc pas surprenant d'identifier, parmi les bactéries fermentantes caséolytiques de nos 50 échantillons de lait cru, les espèces $S$. marcescens $(61 / 62)$ et $E$. cloacae (1/62).

En ce qui concerne les bactéries possédant une oxydase, nous notons la présence d'Aeromonas dans seulement 4 p. 100 des échantillons et, en moyenne, ce genre ne représente que 3,5 p. 100 de la flore psychrotrophe caséolytique.

Certaines espèces d'Aeromonas ont la faculté de se développer à $1^{\circ} \mathrm{C}$ et les 79 souches identifiées par Thomas et Druce [115] sont caséolytiques et lipolytiques. Dans la plupart des études, ce genre ne constitue que 5 p. 100 ou moins de la flore psychrotrophe des laits crus réfrigérés : 3,5 p. 100 [81], 3,1 p. 100 [23], 2,5 p. 100 [89], 2,4 p. 100 [21]; seul Dempster [23] rapporte une teneur de 6,3 p.100.

Présents seulement dans 4 échantillons, nous les identifions à A. caviae (23/35), à A. hydrophila $(7 / 35)$ et à $A$. punctata $(5 / 35)$. Pour leur part, Kielwein et al. [55] indiquent leur présence dans 17 p. 100 des laits crus et les identifient à $A$. hydrophila var, anaerogenes $(41 / 57)$ et à $A$. hydrophila $(16 / 57)$, tandis que Kleeberger n'isole que $A$. punctata [60]. 


\subsection{BACTÉRIES OXYDANTES}

Les bactéries qui dégradent le glucose par voie oxydative représentent 85,8 p. 100 de la flore caséolytique Gram négative et 95,4 p. 100 d'entre elles élaborent soit de la pyoverdine $(33,3 \mathrm{p} .100)$, soit un pigment caroténoïde de couleur orange $(62,1$ p. 100$)$.

Les bactéries fluorescentes sont aisément identifiées à l'espèce $P$. fluorescens (groupe I de Shewan [107]). Nous classons également dans cette espèce les 39 bactéries apigmentées, car bien qu'incapables de produire de la pyoverdine sur gélose au lait et sur King B, elles possèdent les mêmes caractères biochimiques que $P$. fluorescens (bactérie psychrotrophe, caséolytique, ne se développant pas à $41^{\circ} \mathrm{C}$ $[6,101,108,109])$. Dans notre étude, ces souches apigmentées ne constituent que 12 p. 100 de l'espèce $P$. fluorescens. Un pourcentage identique est mentionné par Stanier et al. [108], et Pickett et Pedersen [98] en identifient 18 p. 100. La production de pyoverdine semble être un caractère variable de cette espèce [17] qui peut être modifié par la composition du milieu de culture [101].

La majorité de nos souches de $P$. fluorescens $(323 / 324)$ ne dégradent pas les nitrates, alors que 86 p. 100 des cultures étudiées par Rhodes [101] montrent cette activité, et les 80 souches classées dans le biotype $\mathrm{G}$ ou $\mathrm{M}$ ne diffèrent du biotype I que par leur inaptitude à produire du levane.

Presque toutes les études indiquent que, parmi les bactéries Gram négatives qui constituent la flore psychrotrophe dominante du lait cru réfrigéré, le genre Pseudomonas occupe la première place [11, $16,39,46,48,50,61,82,83,88,113,114,122,130]$. La proportion de ce genre dans les échantillons est variable, soit supérieure à 80 p. 100 [56, $58,76,86]$, soit entre 60 et 80 p. $100[52,62,106]$, soit entre 50 et 60 p. $100[12,84,89,90,104]$, soit comprise entre 40 et 50 p. $100[10,11$, $23,85]$. Seulement dans quelques travaux, les Pseudomonas, toujours présents, ne constituent plus qu'une faible proportion de la flore psychrotrophe [27, 57, 103].

Dès 1888, l'activité protéolytique du genre Pseudomonas est mise en évidence par Kirchner et Orla-Jensen, cités par Thomas et Druce [115]. Sur 514 souches de Pseudomonas étudiées par Bockelman [10], 85 p. 100 sont protéolytiques et 60 p. 100 liquéfient la gélatine ; 84 p. 100 des micro-organismes protéolytiques isolés par Kiuru [58] sont également des Pseudomonas. Dans ce genre, le groupe des fluorescents domine. $P$. fluorescens est la principale espèce psychrotrophe caséolytique du lait cru [51] et 95 p. 100 des souches de cette espèce, étudiées par Samagh et Cunningham [103] liquéfient la gélatine et produisent une protéolyse sur lait écrémé aux basses températures. Cependant, dans quelques travaux, cette espèce ne représente seulement que 36,6 p. 100 et 28,5 p. 100 des Pseudomonas isolés [23, 114].

Dans la flore caséolytique psychrotrophe de nos 50 échantillons, ce genre, trouvé dans $2 / 3$ des prélèvements, avec une proportion variant 
de 0 à 100 p. 100 , ne domine pas et ne représente seulement que 33 p. 100 des souches identifiées. Toutes sont des $P$. fluorescens, seule espèce caséolytique, psychrotrophe, à pouvoir exclusivement oxydant.

En revanche, les bactéries élaborant un pigment caroténoïde de couleur orange constituent la flore dominante de nos laits crus; elles représentent 62,1 p. 100 des bactéries Gram négatives ou 53,3 p. 100 de la flore psychrotrophe caséolytique. Absentes dans 7 échantillons, elles constituent la totalité de la flore caséolytique de 10 laits.

Pour Abd-el-Malek et Gibson [1], le genre Flavobacterium est activement caséolytique ; Kielwein et Geringer [54] ne le considèrent pas comme étant psychrotrophe vrai, car il présente une croissance lente entre 4 et $8^{\circ} \mathrm{C}$. Pour un Flavobacterium dont la température optimale de développement est de $25^{\circ} \mathrm{C}$, Lembo [66] calcule un temps de génération de $20 \mathrm{~h}$ ou plus, à $0^{\circ} \mathrm{C}$, et $47 / 60$ des souches de Flavobacterium, étudiées par Druce (Thomas [112]), forment des colonies visibles en $14 \mathrm{j}$ entre 3 et $5^{\circ} \mathrm{C}$; Lawton et Nelson [63] isolent même, de lait cru stocké à $3^{\circ} \mathrm{C}$, un Flavobacterium psychrophile obligatoire ayant un optimum de température voisin de $10^{\circ} \mathrm{C}$.

Ces bactéries représentent généralement entre 0 et 20 p. 100 de la flore aérobie psychrotrophe du lait cru réfrigéré $[3,23,48,58,62,73$, $76,84,85,89,91,106,114]$. Bien que Kielwein et Geringer [53] notent leur présence dans 86,9 p. 100 et 85,7 p. 100 des prélèvements en été et en hiver, ce genre ne constitue seulement que 3,49 p. 100 de la flore banale.

Nous avons rencontré quelques difficultés à identifier nos souches pigmentées en orange au genre Flavobacterium, défini par Weeks [128], car aucune ne correspond en tous points aux 12 espèces répertoriées dans ce genre, comme Geringer et Kielwein [33], en 1975, l'avaient déjà constaté. De nombreux essais de classification fondés sur la mobilité, l'aptitude à se mouvoir par déformation de la paroi cellulaire $[29,67,96]$, le contenu en A.D.N. (CG p. 100) $[32,41,79]$, traduisent la très grande hétérogénéité taxonomique de ce groupe de bactéries $[67,127]$, dont certaines espèces appartiendraient au genre Cytophaga. C'est ainsi que Gavini et Leclerc [32] différencient les espèces à CG p. 100 bas (27,1 p. $100-39$ p. 100) (Cytophaga), des espèces ayant un CG p. 100 élevé $(52,7-63,1)$ (Flavobacterium).

Nos 531 souches pigmentées en orange possèdent en commun un certain nombre de caractères phénétiques constamment positifs ou négatifs.

- Caractères positifs : catalase ; oxydase ; cytochrome-oxydase ; croissance à $30^{\circ} \mathrm{C}$ et à $7^{\circ} \mathrm{C}$; développement en milieu à $2 \mathrm{p} .100$ en $\mathrm{NaCl}$; résistance à la polymyxine $\mathrm{B}$ en disques à $50 \mathrm{U}$, dégradation du glucose, du d-lévulose, du mannose (en milieu liquide exempt de peptone) ; présence de gélatinase, de phosphatase, de lipase (Tween 80) ; d'estérase (tributyrine). 
- Caractères négatifs : immobilité, pas de mobilité par glissement; pas de croissance à $41^{\circ} \mathrm{C}$ et en milieu à 3 p. 100 en $\mathrm{NaCl}$; pas de développement en milieu de Mac Conkey (agar-glucose) ; pas d'utilisation du citrate de $\mathrm{Na}$, pas de production de levane; absence de nitrate-réductase, d'A.D.H., de L.D.C., d'O.D.C., de DNase, d'activité cellulolytique.

- Certains caractères importants pour leur identification sont variables ; tryptophane désaminase $(v+, 75$ p. 100), $\beta$-galactosidase $(\mathrm{v}+, 53 \mathrm{p} .100)$, uréase $(\mathrm{v}-, 17 \mathrm{p} .100)$, lécithinase $(\mathrm{v}+, 76 \mathrm{p} .100)$, phosphatase $(\mathrm{v}+, 75$ p. 100), amylase $(\mathrm{v}+, 75$ p. 100).

L'utilisation du milieu de Hugh et Leifson, pour l'étude du mode de dégradation des sucres, donne des résultats ininterprétables, car ces souches étant peu glucidolytiques, l'acidification peut être masquée par la présence de peptones, même en faible concentration. En général, ces souches acidifient très légèrement, après 24 h d'incubation, le milieu de Hugh et Leifson en contact avec l'air, puis montrent une attaque discrète, après 4 à $5 \mathrm{j}$, du glucose en anaérobiose [19]. Gavini et Leclerc [32] indiquent que $2 / 3$ des bactéries l'oxydent et que $1 / 3$ sont alcalinisantes ou inertes, résultat voisin du nôtre : oxydantes 57,5 p. 100 , inertes 1,5 p. 100 , alcalinisantes 41 p. 100 .

La caractérisation d'un CG p. 100* bas, compris entre 33 et 34 p. 100, nous permet de tenter un classement de nos souches d'après les travaux de taxonomie numérique effectués depuis une dizaine d'années.

Nous pouvons inclure toutes nos souches dans le pleista B, caséolytique, de Bean et Everton [8], Everton et al. [28], mais sa subdivision en 5 sous-pleista ne nous permet pas de conclure valablement. D'abord classées pour 97 p. 100 d'entre elles dans le groupe II de Pickett et Manclark [97], Pickett et Pedersen [99, 100], groupe saccharolytique assimilable au groupe II B de King, nous les en retirons toutes, sur la base des travaux de Owen et Lapage [92], Owen et Snell [93], car aucune ne produit de $\mathrm{l}^{\prime} \mathrm{H}_{2} \mathrm{~S}$. Le groupe $\mathrm{O}$ de Mc Meekin et al. [71] ne peut également convenir, car elles ne possèdent pas d'A.D.H., mais nous pouvons classer la majorité de nos souches (75 p. 100) dans le groupe I, établi en 1972 [70], par ces mêmes chercheurs. Malgré l'absence, dans la classification, de caractères aussi importants que la mobilité ou l'élaboration d'une oxydase, nous les assimilons aux 2 sous-pleista II II $_{0}$ et II de Byrom [20]. Toutes sont identifiées aux bactéries saccharolytiques de Gilardi $[34,36]$, au genre Cytophaga défini par Gavini et Leclerc [32], et au phénon 1 de Hayes [42].

* La détermination du CG p. 100 a été réalisée par le service du professeur Leclerc à l'I.N.S.E.R.M. de Villeneuve-d'Ascq. 
Toutes ces classifications sont encore sujettes à de nombreuses controverses et modifications ; en 1977, Hayes [42] propose pour les bactéries immobiles possédant un CG p. 100 bas, "Cytophaga atypiques ", identifiées au genre Cytophaga de Gavini et Leclerc [32], de nouvelles définitions des genres Flavobacterium et Flexibacter, et pour celles ayant un CG p. 100 élevé (mobiles ou immobiles), le genre Empedobacter (Brisou [15]).

\subsection{BACTÉRIES ALCALINISANTES}

Seules 11 souches, issues de 7 échantillons de lait, sont identifiées au genre Acinetobacter et plus particulièrement à A. calcoaceticus, seule espèce mentionnée dans la $8^{c}$ édition du Manuel de Bergey [22] qui regroupe des bactéries classées auparavant dans le genre Achromobacter, non protéolytique [6]. Le genre Acinetobacter, immobile et dépourvu d'oxydase [44], élabore des lipases, pour plus de 90 p. 100 des souches, mais très peu de bactéries, moins de 15 p. 100, présentent une activité protéolytique [7].

\section{CONCLUSION}

La flore caséolytique psychrotrophe de nos 50 échantillons de lait cru réfrigéré est essentiellement constituée d'espèces Gram négatives (96,7 p. 100), mais contrairement à la plupart des recherches, le genre Pseudomonas, et plus particulièrement l'espèce $P$. fluorescens, ne dominent pas. Les bactéries pigmentées, élaborant un pigment diffusible ou non, P. fluorescens, Cytophaga, Micrococcus, sont souvent isolées $(83,8$ p. 100). L'importance des espèces élaborant un pigment caroténoïde de couleur orange semble être liée à la saison, car les prélèvements étant effectués au mois de mai, Andrey et Frazier [3] observent que, lorsque les vaches sont en pâture, le genre Flavobacterium constitue 56 p. 100 de la flore du lait cru stocké à $3,3^{\circ} \mathrm{C}$. Ce genre, à taxonomie incertaine, est caractéristique de l'eau $[8,121]$ et est également identifié dans diverses analyses d'herbes [25].

En plus des différentes sources de contamination, telles que le sol, l'eau et la nourriture, la prédominance de certains genres bactériens est liée à l'état sanitaire de l'équipement laitier ; il n'est donc pas surprenant de noter de telles différences dans la composition de la flore psychrotrophe caséolytique des laits crus, d'une étable à l'autre. Il convient donc d'améliorer l'hygiène de la traite et d'entraver au maximum, pendant le stockage en tank, la prolifération des bactéries caséolytiques psychrotrophes, par une réfrigération peu prolongée de 24 à $48 \mathrm{~h}$, à des températures inférieures ou égales à $3^{\circ} \mathrm{C}$, car ces bactéries, thermolabiles, élaborent des protéases, non totalement inactivées par des traitements thermiques tels que 1'U.H.T., qui peuvent être à l'origine, dans certains laits fortement contaminés, de défauts de flaveur et de texture. 


\section{Ré s u m é}

996 souches, représentant la flore caséolytique psychrotrophe, sont isolées de 50 échantillons de laits crus, prélevés au cours du mois de mai 1975 au sud-ouest de Nancy et stockés en tanks à la ferme pendant 24 à $48 \mathrm{~h}$ entre 3 et $5^{\circ} \mathrm{C} .96,7 \mathrm{p} .100$ des souches sont identifiées à des espèces Gram négatives ; 84 p. 100 élaborent soit un pigment diffusible, soit un pigment de nature caroténoïde. Les bactéries pigmentées de couleur orange, qui possèdent les mêmes caractères génomiques et phénétiques que les Cytophaga de Gavini et Leclerc (1975), constituent la flore dominante (53,3 p. 100). Les autres souches sont identifiées à $P$. fluorescens (32,5 p. 100), S. marcescens et $E$. cloacae (6,2 p. 100), Aeromonas caviae, A. hydrophila, A. punctata $(3,5$ p. 100), M. varians et M. Luteus (3,3 p. 100) et à Acinetobacter calcoaceticus (1,1 p. 100).

Compte tenue de la rareté des publications consacrées à l'identification de la flore caséolytique psychrotrophe des laits crus réfrigérés, les auteurs comparent leurs résultats avec ceux concernant la composition de la flore aérobie psychrotrophe.

\section{S u $\mathrm{m} \mathbf{m}$ a r y}

996 strains constituting caseolytic psychrotrophic flora were isolated from 50 samples of raw milk, collected during the month of May 1975 in the south-west of Nancy and stored in bulk tanks for 24 to $48 \mathrm{~h}$ between $3^{\circ} \mathrm{C}$ and $5^{\circ} \mathrm{C}$.

96.7 p. 100 of the strains were identified as Gram-negative species. 84 p. 100 produced either a diffusible pigment or a carotenoid pigment. The orange-coloured bacteria, which possessed the same genomic and phenetic characters as the Cytophaga of Gavini and Leclerc, constituted the dominant flora (53.3 p. 100).

The other strains were identified as $P$. fluorescens ( 32.5 p. 100), S. marcescens and E. cloacae (6.2 p. 100), A. caviae, A. hydrophila, A. punctata (3.5 p. 100), M. varians and M. luteus (3.3 p. 100) and as Acinetobacter calcoaceticus (1.1 p. 100).

Given the scarcity of publications devoted to the identification of the caseolytic psychrotrophic flora of refrigerated raw milks, the authors compare their results with those concerning the composition of the aerobic psychrotrophic flora. 


\section{Bibliographie}

[1] Abd-el-Malek (Y.), Gibson (T.) (1952). - J. Dairy Res., 19, 3, 294-301.

[2] American Public Health Association (1967). - Standard methods for the examination of dairy products. New York, Am. Publ. Hlth. Ass., 12th ed., $304 \mathrm{p}$.

[3] Andrey (J.), Frazier (W. C.) (1959). - J. Dairy Sci., 42, 11, 1781-1784.

[4] Arnold (D. R.), Murphy (R. G.) (1975). - Abstr. Ann. Meeting Amer. Soc. Microbiol., 75, 276

[5] Baird-Parker (A. C.) (1963). - J. Gen. Microbiol., 30, 3, 409-427.

[6] Barnes (E. M.), Mriton (W.) (1971). - J. Appl. Bact., 34, 599-609.

[7] Baumann (P.), Doudoroff (M.), Stanier (R. Y.) (1968). - J. Bact., 95, 5, 1520-1541.

[8] Bean (P. G.), Everton (J. R.) (1969). - J. Appl. Bact., 32, 1, 51-59.

[9] Bloouel (R.) (1974). - D.E.A. d'Agronomie, Université Nancy I, 83 p.

[10] Bockelmann (I. von) (1966). - Milchwissenschaft, 21, 5, 275-278.

[11] Bockelmann (I. von) (1969). - Meddn. Svenska Mejeriern. Riksfören, 91, 15 p., Anal.: Dairy Sci. Abstr., 31, 12, abstr. 4491.

[12] Bockelmann (I. von) (1970). - XVIII ${ }^{\mathrm{e}}$ Congr. Int. Lait., 1 F, 109.

[13] Bockelmann (I. von) (1974). - XVIII ${ }^{e}$ Congr. Int. Lait., $1 F$, 440.

[14] BrandL (E.) (1968). - Die Molkerei-Ztg, 22, 33, 1109-1114.

[15] Brisou (J.) (1957). - Ann. Inst. Pasteur, 93, 397-404.

[16] Busse (M.) (1965). - Dtsche Molkerei-Ztg, 86, 45, 1777-1781.

[17] Buttiaux (R.) (1961). - Ann. Inst. Pasteur, 101, suppl. $\mathrm{n}^{\circ}$ 6, 43-58.

[18] Buttiaux (R.), Beerens (H.), Taquet (A.) (1974). - Manuel de techniques bactériologiques. $4^{e}$ éd. Flammarion Médecine-Sciences, Paris $6^{\mathrm{e}}, 700 \mathrm{p}$.

[19] Buttiaux (R.), Vandepitte (J.) (1960). - Ann. Inst. Pasteur Lille, 98, 3, 398-404.

[20] Byrom (N. A.) (1971). - J. Appl. Bact., 34, 2, 339-346.

[21] Coujard (C.) (1971). - D.E.A. d'Agronomie, Université Nancy I.

[22] Cowan (S. T.), Holt (J. G.), Liston (J.), Murray (R. G. E.), Niven (C. F.), RAvin (A. W.), Stanier (R. Y.) (1974), - Bergey's manual of determinative bacteriology, 8th ed., Buchanan (R. E.) and Gibbons (N. E.) ; co-editors, The Williams and Wilking Company, Baltimore.

[23] Dempster (J. F.) (1968). - J. Appl. Bact., 31, 3, 290-301.

[24] Desmazeaud (M.), Hermier (J.) (1968), - Ann. Biol. Anim. Bioch. Bioph., 8, 4, 565-577.

[25] Druce (R. G.), Thomas (S. B.) (1970). - J. Appl. Bact., 33, 2, 420-435.

[26] Durr (R.) (1974). - Rev. Lait. franç., n 236, 913-919.

[27] Erdman (I. E.), Thornton (H. R.) (1951), - Can J. Tech., 29, 232-237.

[28] Everton (J. R.), Bean (P. G.), Bashford (T. E.) (1968). - J. Fd Techn., 3, 241-247.

[29] Floodgate (G. D.), Hayes (P. R.) (1963). - J. Gen. Microbiol., 30, 237-244.

[30] Forbes (D.) (1968), - J. Appl. Bact., 31, 4, 426-435.

[31] Foster (E. M.), Nelson (F. E.), Speck (M. L.), Doetsch (R. N.), Olson (J. C.) (1957). - Dairy microbiology. Prentice-Hall, Inc., Englewood Cliffs, New Jersey, $492 \mathrm{p}$. 
[32] Gavini (F.), Leclerc (H.) (1975). - Rev. Int. Océanogr. Méd., 37-38, 17-67.

[33] Geringer (M.), Kielwein (G.) (1976). - Archiv für Lebensmittel-Hyg., 27, $1,11-17$.

[34] Gilardi (G. L.) (1974). - (Note non publiée).

[35] Gilardi (G. L.) (1975). - Health Lab. Sci., 12, 4, 311-315.

[36] Gilardi (G. L.) (1976). - (Note non publiée), revised january, 1-8.

[37] Graham (D. C.), Hodgkiss (W.) (1967). - J. Appl. Bact., 30, 1, 175-189.

[38] Greene (V. W.), Jezeski (J. J.) (1954). - Appl. Microbiol., 2, 110-117.

[39] Grün (L.), Konstantinidou (A.) (1971). - Milchwissenschaft, 26, 7, 413-415.

[40] Hayes (P. R.) (1963). - J. Gen. Microbiol., 30, 1, 1-19.

[41] Hayes (P. R.), Wilcock (A. P. D.) (1977). - J. Appl. Bact., 43, 111-115.

[42] Hayes (P. R.) (1977). - J. Appl. Bact., 43, 345-367.

[43] Hechelmann (H.), Leistner (L.) (1969). - Archiv für Lebensmittel-Hyg., 20, $8,169-179$.

[44] Hendrie (M. S.), Hodgkiss (W.), Shewan (J. M.) (1964), - Ann. Inst. Pasteur Lille, 15, 43-60.

[45] Hendrie (M. S.), SHewan (J. M.) (1966). - Identification methods for microbiologists. Ed. by Gibbs (B. M.), Skinner (F. A.) Part A. Academic Press, London, New York, 1-6, $145 \mathrm{p}$.

[46] Higoshi (H.) (1964). - Jap. J. Vet. Res., 12, 2, 33, Anal.: Dairy Sci. Abstr., 27, 4, 171, abstr. 1182 .

[47] Hugh (R.), Leifson (E.) (1953). - J. Bact., 66, 25-26.

[48] Jayashankar (S. R.), Dudani (A. T.), IYa (K. K.) (1966). - XVII ${ }^{\text {e }}$ Congr. Int. Lait., $B$, 539-547.

[49] Johns (C. K.) (1959). - J. Dairy Sci., 42, 10, 1625-1650.

[50] Juffs (H. S.) (1973). - J. Appl. Bact., 36, 4, 585-598.

[51] Juffs (H. S.) (1974). - Aust. J. Dairy Techn., 29, 2, 74-78.

[52] Kielwein (G.), (1968). - Archiv Lebensmittel-Hyg., 19, 2, 25-30.

[53] Kielwein (G.), Geringer (M.) (1975). - Milchwissenschaft, 30, 10, 608-609.

[54] Kielwein (G.), Geringer (M.) (1975). - Archiv Lebensmittel-Hyg., 26, 6, 221-225.

[55] Kielwein (G.), Gerlach (R.), Johne (H.) (1969). - Archiv LebensmittelHyg., 20, 2, 34-38.

[56] Kikuchi (M.), Matsui (Y.) (1974). - Jap. J. Zootechn. Sci., 45, 11, 592-596, Anal.: Dairy Sci. Abstr., 37, 7, 412, abstr. 4319.

[57] KIURU (K.), EKLUND (E.) (1970). - Karjantuote, 53, 4, 124-126.

[58] Kiuru (K.), Eklund (E.), Gyllenberg (H.), Antila (M.) (1971). - Milchwissenschaft, 26, 3, 138-141 et XVIII ${ }^{\mathrm{e}}$ Congr. Int. Lait., 1 F, 111 (1970).

[59] KleEberger (A.) (1975). - Milchwissenschaft, 30, 10, 602-603.

[60] KleEBerger (A.) (1977). - Z. Lebensm. Unters.-Forsch., 163, 1, 44-47.

[61] Kundrat (W.) (1966). - Archiv Lebensmittel-Hyg., 17, 7, 160-162.

[62] Lagrange (W. S.), Nelson (F. E.) (1961). - J. Dairy Sci., 44, 8, 1440-1445.

[63] Lawton (W. C.), Nelson (F. E.) (1954). - J. Dairy Sci., 37, 10, 1164-1172.

[64] Leclerc (H.) (1961). - Thèse d'Etat Pharmacie, $n^{\circ}$ 272, Lille.

[65] Lelliot (R. A.), Billing (E.), Hayward (A. C.) (1966). - J. Appl. Bact., 29, $3,470-489$. 
[66] Lembo (M.) (1968). - Riv. Ist. Sieroter. Italiano, 43, 6, 308-313.

[67] Lewin (R. A.) (1969). - J. Gen. Microbiol., 58, 189-206.

[68] Lysenko (O.) (1961). - J. Gen. Microbiol., 25, 379-408.

[69] Mc Kenzie (D. A.), Egdell (J. W.), Clegg (L. F. L.), Thomas (S. B.) (1949). XII ${ }^{\mathrm{e}}$ Congr. Int. Lait, 2, 2, 536-545.

[70] Mc Meekin (T. A.), Stewart (D. B.), Murray (J. G.) (1972). - J. Appl. Bact., $35,1,129-137$.

[71] Marth (E. H.), Frazier (W. C.) (1957). - J. Milk Fd Techn., 20, 3, $72-78$.

[72] Marth (E. H.), Frazier (W. C.) (1957). - J. Milk Fd Techn., 20, 3, 79-83.

[73] Marth (E. H.), Frazier (W. C.) (1957). - J. Milk Fd Techn., 20, 4, 93-99.

[74] Martley (F. G.), Jayashankar (S. R.), Lawrence (R. C.) (1970). - J. Appl. Bact., 33, 363-370 et XVIII ${ }^{\text {e }}$ Congr. Int. Lait., 1 F, 157.

[75] Mikawa (K.), Hoshino (T.) (1973), - Jap. J. Dairy Sci., 22, 5, A 176-A 186.

[76] Millière (J. B.), Ben Rachid (S. M.), Chadli (A.) (1973). - Archiv. Inst. Pasteur, Tunis, 50, 189-210.

[77] Millière (J. B.) (1978). - Thèse de Docteur-Ing., Université Nancy I.

[78] Millière (J. B.), Veillet-Poncet (L.) (1979). - Rev. Lait. Franç, 331, 61-67.

[79] Mitchell (T. G.), Hendrie (M. S.), Shewan (J. M.) (1969). - J. Appl. Bact., 32, $1,40-50$.

[80] Møller-Madsen (A.), Hansen (K) (1967). - Beretn. St. For Øgsmejeri, 167, 32 p., Anal.: Dairy Sci. Abstr., 32, 6, 346, abstr. 2394.

[81] Morse (P. M.), Jackson (H.), Mc Naughton (C. H.), Legatt (A. G.), Landerkin (G. B.), Johns (C. K.) (1968). - J. Dairy Sci., 51, 8, 1182-1190.

[82] Mrozek (H.) (1970). - XVIII Congr. Int. Lait., 1 F, 512.

[83] Murthy (L.), Herreid (E. O.), Mc Whitney (L. R.) (1958), - J. Dairy Sci., $41,10,1324-1341$.

[84] NaKAE (T.) (1970). - Milchwissenschaft, 25, 3, 161-167.

[85] NaKanishi (T.), NAKAE (T.), RASAN (B. S.) (1967). - Tohuku J. agric. Res., 18, 4, 247-255, Anal.: Dairy Sci. Abstr., 30, 10, 547, abstr. 3576.

[86] Nakanishi (T.), Tanabe (T.) (1970). - Jap. J. Dairy Sci., 19, 2, A 44-A 50.

[87] Newton (S. B.) (1965). - Dissert. Abstr., 26, 1, 374.

[88] Ogawa (M.) (1967). - Jap. J. Dairy Sci., 16, 6, A 168-176.

[89] Ogawa (M.) (1967). - Jap. J. Dairy Sci., 16, 6, A 177-184.

[90] OrR (M. J.), Mc LaRty (R. M.), Mc Cance (M. E.), Baines (S.) (1964). Dairy Ind., 29, 3, 169-173.

[91] Otenhajmer (I.), Mitic (S.) (1971). - Mljekarstvo, 21, 4, 74-79, Anal.: Dairy Dairy Sci. Abstr., 33, 10, 788, abstr. 5239.

[92] Owen (R. J.), Lapage (S. P.) (1974), - Antonie van Leeuwenhoek, 40, 2 , 255-264.

[93] OWen (R. J.), SNell (J. J. S.) (1973). - Antonie van Leeuwenhoek, 39, 3, 473-480.

[94] Panes (J. J.), Thomas (S. B.) (1959). - J. Appl. Bact., 22, 272-277.

[95] Panes (J. J.), Thomas (S. B.) (1968). - J. Appl. Bact., 31, 4, 420-425.

[96] Perry (L. B.) (1973). - J. Appl. Bact., 36, 2, 227-232.

[97] Pickett (M. J.), Manclark (C. R.) (1970). - Amer. J. Clin. Path., 54, 2, 155-163.

[98] Pickett (M. J.), Pedersen (M. M.) (1968). - Appl. Microbiol., 16, 1631-1632. 
[99] Pickett (M. J.), Pedersen (M. M.) (1970). - Can. J. Microbiol., 16, 351-362.

[100] Pickett (M. J.), Pedersen (M. M.) (1970). - Amer. J. Clin. Path., 54, 2, 164-177.

[101] Rhodes (M. E.) (1959). - J. Gen. Microbiol., 21, 221-263.

[102] Salisbury (W. A.), Likos (J. J.) (1972). - J. Clin. Path., 25, 12, 1083-1085.

[103] Samagh (B. S.), Cunningham (J. D.) (1972). - J. Dairy Sci., 55, 1, 19-24.

[104] Schultze (W. D.), Olson (J. C. Jr) (1957). - J. Dairy Sci., 40, 6, 602, abstr. M. 23.

[105] Schultze (W. D.), Olson (J. C. Jr) (1960). - J. Dairy Sci., 43, 3, 346-350.

[106] Schultze (W. D.), Olson (J. C. Jr) (1960). - J. Dairy Sci., 43, 3, 351-357.

[107] Shewan (J. M.), Hobbs (G.), Hodgkiss (W.) (1960). - J. Appl. Bact., 23, 379-390.

[108] Stanier (R. Y.), Palleroni (N. J.), Doudoroff (M.) (1966). - J. Gen. Microbiol., 43, 159-270.

[109] SutTer (V. L.) (1968). - Appl. Microbiol., 16, 10, 1532-1538.

[110] TeXdorf (I.), Kielwein (G.), Ergüllü (E.) (1976). - Archiv LebensmittelHyg., 26, 2, 46-48.

[111] Thomas (S. B.) (1955). - J. Appl. Bact., 18, 2, 331-357.

[112] Thomas (S. B.) (1958). - Dairy Sci. Abstr., 20, 5, 357-370 (Part I) and 20, 5, $447-468$ (Part II).

[113] Thomas (S. B.) (1974). - Dairy Ind., 39, 7, 237-240 and 39, 8, 279-282.

[114] Thomas (S. B.), Druce (R. G.) (1963). - Dairy Engng., 80, 10, 378-381.

[115] Thomas (S. B.), Druce (R. G.) (1971). - Dairy Ind., 36, 2, 145-150.

[116] Thomas (S. B.), Druce (R. G.) (1972). - Dairy Ind., 37, 11, 593-598.

[117] Thomas (S. B.), Hobson (P. M.), Bird (E. R.) (1959), - XVe Congr. Int. Lait., $3,5,1334-1340$,

[118] Thomas (S. B.), King (K. P.), Jones (T. I.), Griffiths (D. G.) (1965). - Dairy Ind., 30, 1, 41-45.

[119] Thomas (S. B.), Lewis (E.) (1957). - J. Appl. Bact., 20, 1, 11-16.

[120] Thomas (S. B.), Sekhar (C. V.) (1946). - Proc. Soc. Appl. Bact., 1, 47-50, Anal.: Dairy Sci. Abstr., 9, 129.

[121] Thomas (S. B.), Thomas (B. F.) (1947). - Proc. Soc. Appl. Bact., 2, 65-69, Anal.: Dairy Sci. Abstr., 11, 31.

[122] Thomas (S. B.), Thomas (B. F.) (1973). - Dairy Ind., 38, 1, 11-15 (Part I) and $38,2,61-70$ (Part II).

[123] Thornley (M. J.) (1960). - J. Appl. Bact., 23, 37-52.

[124] Veillet-Poncet (L.) (1973). - Thèse Doctorat Sc. Nat., Université Nancy I. Le Lait, 54, 537, 409-414 ; 538, 537-552 ; 539-540, 675-684.

[125] Watrous (G. H.), Doan (F. J.), Josephson (D. V.) (1952). - Bull. Pa. Agric. Exp. Sta., 551, Anal.: Dairy Sci., Abstr., 14, 9, 785.

[126] WeEKS (O. B.) (1955). - J. Bact., 69, 649-658.

[127] WeEKS (O. B.) (1969). - J. Appl. Bact., 32, 13-18.

[128] WeEKs (O. B.) (1974). - In Bergey's Manual of Determinative Bacteriology 8th edn, ed. Buchanan R. E. and Gibbons N. E., Baltimore: Williams and Wilkins Co.

[129] Wilssens (A. T. E.), Casteele (J. C. van de), De Meter (R. L.) (1966). - XVII ${ }^{\circ}$ Congr. Int. Lait., $B, 431-434$.

[130] Yano (N.), Morichi (T.) (1974). - Bull. Nat. Inst. Anim. Ind., 28, 41-45, Anal.: Dairy Sci. Abstr., 39, 1, 56, abstr. 431. 